\title{
Repeated jump ability of young basketball players at different game stages and its relationship to aerobic capacity
}

\author{
Shai Segev a and Yoav Meckel a, ${ }^{*}$
}

a Life Science Department, The Academic College at Wingate, Wingate Institute, Netanya 4290200, Israel

*Corresponding author Email: meckel@wincol.ac.il

DOI: https://doi.org/10.34256/ijpefs2034

Received: 01-05-2020, Revised: 25-07-2020; Accepted: 17-08-2020; Published: 21-09-2020

Abstract: The aim of the study was to determine the performance indices of a repeated jumping test (RJT) over three different stages of a basketball game, and to examine their relationships with the aerobic capacity of young basketball players. Sixteen young (17.2 $\pm 0.4 \mathrm{yrs})$ trained basketball players performed an RJT (six sets of six consecutive vertical jumps) after warm-up, at halftime, and after completing a full game, as well as an aerobic power test (shuttle run test for $20 \mathrm{~m}$ ), each test taking place on a different day. Performance indices for each of the RJTs were the ideal jump height (IJ), the total jump height (TJ) of all the jumps, and the performance decrement (PD) throughout the tests. The IJ and TJ were significantly higher at the halftime compared with both after warmup and after a full-time game $(p<0.01)$. No major variations were noted in IJ and TJ in the full-time game compared to the warm-up. During any of the three game stages, there was no significant difference in the PD. No significant relationships were found between the aerobic capacity and any of the RJT performance indices at the different game stages. Given the present findings, coaches and players may consider the use of a more intense warm-up protocol, one that will efficiently prepare players for the early stages of a basketball game. The results also suggest that the aerobic energy system's involvement with repeated jumping activity is only minor when young players play basketball.

Keywords: Anaerobic capabilities, Fatigue, Warm-up, Sprint, Recovery
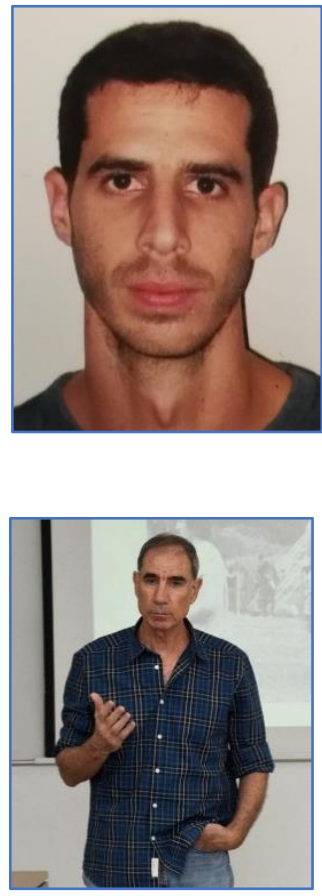

Shai segev is a basketball coach and organization administrator at Maccabi Tel Mond basketball club. He has been working as a physical education teacher since 2015. Research interest includes physiological indices and physical fitness. Shai also teaches basketball courses at the Wingate Academic College.

Dr. Yoav Meckel is a professor of exercise physiology and currently serves as the head of the graduate school at the Academic College at Wingate, Israel. He has published over 100 articles focusing on different aspects of athletic performance and training. In recent years professor Meckel has been involved in research that focus on human genetics and their effect on athletic performance.

\section{Introduction}

Male basketball players have previously been studied by activity patterns and physiological responses during competition. For example, Ben Abdelkrim, EI Fazaa \& El Ati [1] found that the frequency of all activities was $1050 \pm 51$ for young (under 19 yrs) players, with guards performing the highest number of activities (1103 \pm 32 ), while centers and forwards performing $1026 \pm 27$ and $1022 \pm 50$ activities during the game, respectively. In another study, Klusemann, Pyne, Hopkins \& Drinkwater [2] reported that the frequency of all activities was $809 \pm 80$ for young national league players (age $17.8 \pm 0.6 \mathrm{yrs}$ ) during basketball games. Previously, McInnes, Carlson, Jones \& McKenna [3], found the frequency of all activities during a game to be $997 \pm 183$, with a change in movement category occurring every two sec. Furthermore, Torres-Ronda et al. [4], found that first division top-level Spanish players performed an average of 33 movements per min. during seven 
basketball matches, and that peak heart rate (HR) was $198 \mathrm{~b} / \mathrm{m}$ (96.8\% of maximal HR). The average HR throughout the games was $158 \mathrm{~b} / \mathrm{m}$ (78\% of maximal $H R)$. These demonstrate the quick and fast actions in basketball games with regular change of players' movement patterns.

It has been well documented that the vertical jump is a crucial activity in basketball, and is executed defensively as well as offensively while rebounding, blocking or shooting the ball $[1,3,5]$. Ben Abdelkrim et al. [1], reported an average of $44 \pm 7$ jumps during a game for elite-level adult basketball players. Similarly, McInnes et al. [3] reported $46 \pm 12$ jumps during a match for Australian national basketball league players. The implications of jumping action in basketball were demonstrated when Scanlan et al. [5] found that elite front-line players jumped an average of $56 \pm 2$ times and back-line players an average of $42 \pm 6$ times during a game. In contrast, in a domestic-level basketball league, front-line and back-line players jumped only $49 \pm 3$ and $41 \pm 3$ times, respectively, during the game.

The RST has become a very popular testing procedure for evaluating the anaerobic capability of team players over the past three decades [6-8]. Such tests involve repetitions of short sprints, with short recovery periods in-between. However, given the importance of jumping in basketball, measuring the ability of players to repeatedly jump throughout the game may be also a relevant method for evaluating players' athletic anaerobic capabilities [9]. To the best of our knowledge, only one study thus far has measured the anaerobic capabilities of team sports players using the repeated jump test [10]. In this study, professional male volleyball players performed six sets of six consecutive maximal jumps, with 30-sec periods rest between sets. It should also be noted that in most studies the ability to repeatedly perform intense activity was measured when players were recovered and after a proper warm-up procedure. Nevertheless, players have to repeatedly produce instance activities throughout the entire game when they may be tired especially at the last stages of the game. Two investigations have assessed the repetitive sprinting capabilities of basketball [8] and soccer [11] players using the standard RST. In these studies, players performed the RST after a warm-up, at halftime and at the end of the game. Given the implications of the players' jumping ability to basketball games, it seems that it would be beneficial to measure this ability at different stages of the game. The aim of the present study was to compare performance indices and physiologic responses following RJTs during different phases of a basketball game - after a warmup, at halftime and at the end of a match. We also looked at the ties between the aerobic fitness of the player and the RJTs' performance indices over three separate stages of a game to investigate the involvement of the aerobic energy system in the repetitive jumping action during the whole game. We thought that the performance indices would decrease and that the physiological responses would increase when the RJT is completed at the halftime and the end of the game compared to after the warm-up. We also suggested that higher aerobic fitness will be associated with higher RJT performance indices at the advanced stages of a game compared to after the warm-up.

\section{Methods}

\subsection{Participants}

Sixteen late-adolescent male basketball players (with age $17.2 \pm 0.4 \mathrm{yrs}$; height $177 \pm 7.0 \mathrm{~cm}$; weight $66.7 \pm 5.9 \mathrm{~kg}$; body fat $12.2 \pm 1.9 \%$ ) participated in the study. In the 20-meter shuttle run test average player aerobic score was $1104 \pm 384 \mathrm{~m}$, determined by the distance achieved. The players trained four days a week and participated in weekends competitions. The study was performed at the last part of the season when players at top physical condition. The training focused on tactical and technical exercises at this stage, while at this time no resistance and aerobic workouts were performed.

For evaluating height and body weight, a regular calibrated scale and stadiometer were used. Four-sites skin-fold measurements have been used to calculate percent body fat, using standard equations (triceps, biceps, sub-scapular and super-iliac). Pubertal status was assessed by Tanner stage for pubic hair, and all the players were late pubertal (Tanner stage 45). Before giving their written informed consent, all participants were informed of the study requirements, benefits and risks. The study was approved by the Ethics Committee for Institutional Research.

\subsection{Procedures}

The participants performed four tests as follow: one aerobic power test, three RJTs at different stages of a basketball game - after a warm-up, at halftime of a game and at the end of a game, each on a separated day. All the tests were performed on a basketball court, in late afternoon, four hours after 
lunch. The coaches were asked to avoid hard work outs the days before testing sessions in order to prevent fatigue among the players.

\subsection{Aerobic Power Test -Shuttle Run Test of 20 Meter}

The test has been shown to be a reliable and valid indicator of aerobic fitness ( $\left.\mathrm{VO}_{2} \max \right)$ [12]. This test is popular among team sport coaches because of the back and forward run that characterizes players' movement pattern during games. The test involves the shuttle runs between two markers that are 20 meters apart at elevated speeds. A portable compact disk (Sony CFD-V7) determined the speed of the run, emitting tones at appropriate intervals. During the test, participants were required to be at one end of the $20 \mathrm{~m}$ course at the signal. After the initial speed of 8.5 $\mathrm{km} /$ hour, speed was increased every minute by 0.5 $\mathrm{km} / \mathrm{hour}$. The score for each participant was decided according to the total distance gained before terminating the run.

\subsection{Repeated Jump Tests}

The three RJTs were separated from one another by three to four days. One test was performed after a warm up, another test at half-time and another test at the end of a game. Six sets of six consecutive maximum vertical jumps, with a rest of $30-\mathrm{sec}$ between sets, comprised each RJT. The six jumps for each set were performed continually without stooping between jumps. In the $30-\mathrm{sec}$ rest between sets, participant walked around for recovery. This protocol was previously described and validated in professional volleyball players [10]. The jumping technique used in each test was the free countermovement jump (FCMJ). Test began in an upright position of the participant. The participant then moved into a semi-squat position, using a vigorous two-arm swing to make a maximum jump. Such jumping technique is usually used repeatedly by players during basketball games. The participants were encouraged verbally at all jumping sets. Jumping was carried out using the Optojump system (Microgait, OGA002, Italy) that recorded jump flight time and height. Each participant made an all-out vertical jump before the performance of the RJT. This jump was used as a criterion for the next test, and at the first jump of the RJT, every participant needed to achieve a minimum of $95 \%$ of his criterion score. The participant was asked to restart the test if such a score was not reached. The criterion score was met by all participants and neither had to restart the test.
One RJT was performed following a typical warm-up procedure (SWT). This procedure consisted of 5 minutes jogging, 5 minutes stretching and 10-15 minutes of basketball running and jumping drills. This warm-up is normally done in most basketball teams by players before matches. At the end of a halftime there was additional RJT and at the end of a full-time basketball match another RJT. Each player performed a halftime test (HTT) after completing a continuous 15 min game, before being quickly taken to the nearby area to perform the RJT. Each player performed an inplay time of fifteen minutes in the first half, ten minutes in rest, and an additional $15 \mathrm{~min}$ in the second half, for the full-time test. About one minute passed for each participant from the end of each activity (warmup, first half and full game) to the start of the RJT. The 15 -min play in each half replicated typical mean playing time for the young basketball players.

The two parts of the game - the first half and the second half - were played under the normal basketball rules, without the coaches calling "time outs." Still, for fouls and penalty shots the game has been stopped. Six additional players (other than the 10 players who started the game) joined the game gradually, in order to enable the 10 starting players to leave gradually the game and to perform the RJTs at the end of the two parts of the game. However, since the six additional players only replaced players who were taken out of the game to perform the RJTs and did not complete a full 15 or 30 min of play, they did not perform the RJTs and did not participate in the study. All the players were asked to play at full intensity as they usually do in any official matches.

At the end of $15 \mathrm{~min}$ and $30 \mathrm{~min}$, two players were taken out of the game and performed the RJT test. The two players jumped the six sets alternately while one player was jumping one set the other was given a $30 \mathrm{sec}$ rest. The players then switched roles and continued the procedure until both completed the six jumping sets. When the two players completed the RJT they returned to the game and replaced two other players who were send to perform the RJT. This procedure continued until the 10 players that originally started the game completed the RJT after the halftime and after the full-time game (each on a different day).

\subsection{Measurements}

Three measurements were collected from each RJT as follow: 1. ideal jump height (IJ), 2. total jump height (TJ), 3. performance decrement (PD). The IJ 
was computed as the highest jump in each set multiplied by six, and TJ as the total of all jumping heights in all sets. The PD was calculated as [(T] / IJ) $X 100]-100$, using as an indication of fatigue. These calculations have been taken from the usual maximal repeated running and cycling tests [6]. A Polar HR monitor (Polar Accurex Plus, Polar Electro, Woodbury, $\mathrm{NY}$ ) was used to measure players HR just before the beginning and immediately at the end of each RJT. The modified Borg scale (1 to 10) [13] was used to determine players rate of perceived exertion (RPE) shortly before the start and at the end of each RJT.

\subsection{Statistical analysis}

In order to ensure normal distribution of the results, the Shapiro-Wilk method has been used. ANOVA for repeated measures with post hoc Bonferroni was used to determine differences in the IJ, $T J, P D, H R$, and the RPE following the RJT after the warm-up, at the halftime, and at the end of the full game. Pearson correlations were computed between the aerobic fitness and the performance indices in the three RJTs. Significance level was set at $p<0.05$. Data are presented as mean $\pm \mathrm{SD}$.

\section{Results}

Means of cardiac response and RPE of the participants before and after the $6 \times 6$ RJT procedure are summarized in table 1 after warm up, at halftime and at full-time basketball. Heart rate prior to the RJT after warm-up was significantly lower compared to after halftime and after the full-time game, as well as after the RJT following warm-up $(p<0.01)$. Heart rate response at the end of the full-time game was significantly higher after the RJT compared to prior to the RJT $(p<0.05)$. RPE prior to the RJT after warm-up was significantly lower compared to after halftime $(p<0.05)$, after the full-time game, and after the RJT following warm-up ( $p<0.01$ for both). Additionally, at halftime and after the full-time game, RPE was greater following the RJT compared to the RPE prior to the RJT ( $p<0.01$ for both) (Figure 1).

The IJ and TJ were significantly higher at the halftime compared to both after warm-up and after the full-time basketball game $(p<0.01)$. No significant differences were found in IJ and $\mathrm{TJ}$ in the full-time game compared to after the warm-up. No significant differences were found in the PD during any of the three game stages.
The relationships between predicted aerobic capacity and RJT performance indices after warm-up, at halftime, and after the full-time game are presented in Table 2. There were no significant relationships between the aerobic capacity and any of the RJT performances indices at the different stages of the game.

\section{Discussion}

The results showed that, compared with the after warming-up RJT performance indices, the RJT performance indices increased substantially after halftime. However, after the full-time game the RJT 's performance indices were significantly lower than the RJT's after half-time, but unchanged compared to the warm-up RJT's performance indices. In addition, no significant relationships were found between the aerobic capacity and any of the RJT performances indices at the different stages of the game. These findings are in contrast to our hypothesis and raise several questions regarding the physiological load and the fatigue process throughout the different stages of a basketball game.

Given the RPE and HR responses after the three stages and the RJTs of this study (see Table 1), it seems that the players experienced greater physiological load and fatigue following the RJTs than they did throughout the different stages of the game itself. These findings seem reasonable, since the time for the jumps in each set of the RJT in the present study was considerably longer ( $\sim \mathrm{sec}$ ) than a single jump or a sprint duration (mean of $1.7 \mathrm{sec}$ ) typically performed by players in basketball [3]. In a typical basketball game, jump frequency is also lower than in the current RJT, with players not making six successive sets of six jumps in each set at any stage of the basketball game [1].

To try to understand the reasons for the considerably higher IJ and TJ performance indices found in the current research after the half time of the game compared to the performance indices after the warm-up stage, we may want to look more carefully at the performance indices after the warm-up, as well as the specific preparation of the players before each of the two RJTs. The warm-up procedure in this study, which players normally perform routinely before a basketball game, lasted about 20-25 minutes. The majority of this warm-up focused on technical drills such as passing, dribbling, and shooting the ball. This indicates that during the warm-up the players 
performed only low-intensity activity that focused on and prepared them mainly for specific basketball skills.

Table 1 Heart rate and RPE responses of the players prior to and following the $6 \times 6$ $\mathrm{RJT}$ procedure at the different game stages (mean $\pm \mathrm{SD}$ ).

\begin{tabular}{|c|c|c|c|}
\hline Parameters & $\begin{array}{c}\text { RJT } \\
\text { Warm-up }\end{array}$ & $\begin{array}{c}\text { RJT } \\
\text { Halftime } \\
\end{array}$ & $\begin{array}{c}\text { RJT } \\
\text { Full-time } \\
\end{array}$ \\
\hline $\begin{array}{l}\text { HR - pre RJT } \\
\text { (beats/min) }\end{array}$ & $136 \pm 25$ & $168 \pm 19 *$ & $167 \pm 9 *$ \\
\hline $\begin{array}{l}\text { HR - post RJT } \\
\text { (beats/min) }\end{array}$ & $168 \pm 15^{*}$ & $174 \pm 12$ & $175 \pm 12^{* *}$ \\
\hline RPE - pre RJT & $2.5 \pm 0.67$ & $3.7 \pm 1.06 * * *$ & $5.1 \pm 1.66^{*}$ \\
\hline RPE - post RJT & $4.8 \pm 1.31 *$ & $6 \pm 1.33 \dagger$ & $7.4 \pm 1.17 \neq$ \\
\hline \multicolumn{4}{|c|}{$\begin{array}{l}* p<0.01 \text { compared to warm-up. } \\
* * p<0.05 \text { compared to HR after a full-time basketball game. } \\
* * * p<0.05 \text { compared to RPE after warm-up. } \\
+p<0.01 \text { compared to RPE at halftime. } \\
\neq p<0.01 \text { compared to RPE after a full-time basketball game. }\end{array}$} \\
\hline
\end{tabular}

Table 2 Relationships between aerobic capacity and repeated jump performance indices at the three game stages.

\begin{tabular}{|l|c|c|c|}
\hline RJT Indices & Warm-up & $\begin{array}{c}\text { Predicted } \mathrm{VO}_{2} \\
\text { Halftime }\end{array}$ & Full-time \\
\hline Ideal jump height $(\mathrm{cm})$ & 0.423 & 0.379 & 0.351 \\
\hline Total jump height $(\mathrm{cm})$ & 0.423 & 0.437 & 0.399 \\
\hline Performance decrement (\%) & -0.061 & -0.362 & -0.273 \\
\hline
\end{tabular}

Indeed, it was found that the players' RPE (2.5 $\pm 0.67)$ and $\mathrm{HR}$ responses $(136 \pm 25 \mathrm{~b} / \mathrm{m})$ were rather low after the warm-up procedure (see Table I). In comparison, it should be pointed out that in sports such as track and field, for example, a standard warmup procedure for a specific event (short sprints, long/high jumps) lasts around 50 to $60 \mathrm{~min}$, and most of it is comprised of intense and powerful muscle work.

It therefore is doubtful whether the lowintensity exercises that comprised the warm-up in the present study can adequately prepare the players' muscles and metabolic systems for the subsequent 36 all-out vertical jumps of the RJT. It may also be possible that such a warm-up is neither long enough nor intense enough to prepare the players for the activity that is expected in the very first minutes of a competitive basketball game. In addition, one more possible reason for the higher RJT performance indices at the halftime compared to the warm-up stage could be that the activity throughout the first half of the game may have served as a better and a more efficient warm-up process than the actual warm-up procedure originally planned for and performed by the players.
However, coaches should consider that if players use the first part of the game as a warm-up stage and are not fully prepared for the beginning of the game, it could make the team vulnerable creating a chance for the opponent team to produce an irreversible lead. Therefore, it seems that coaches and players should consider the use of longer and more intense warm-up procedures, including short sprints and jumping exercises, prior to the beginning of basketball games. Only one study examined the repeated activity indices of players during the entire basketball game [8]. In this study, similar to the findings of the present study, RST $(12 \times 20 \mathrm{~m}$ sprints with $20 \mathrm{sec}$ rest between sprints) performance indices (e.g., ideal sprint time - IT, and total sprint time - TT) were found to be significantly higher at halftime compared to the RST performance indices after the warm-up stage in young (age $17 \pm 0.5$ yrs) basketball players. The researchers in this study concluded that the warm-up procedure most likely was not providing proper physical preparation for the initial stages of the game; therefore, as in the present study, its performance indices were inferior to the halftime performance indices. 


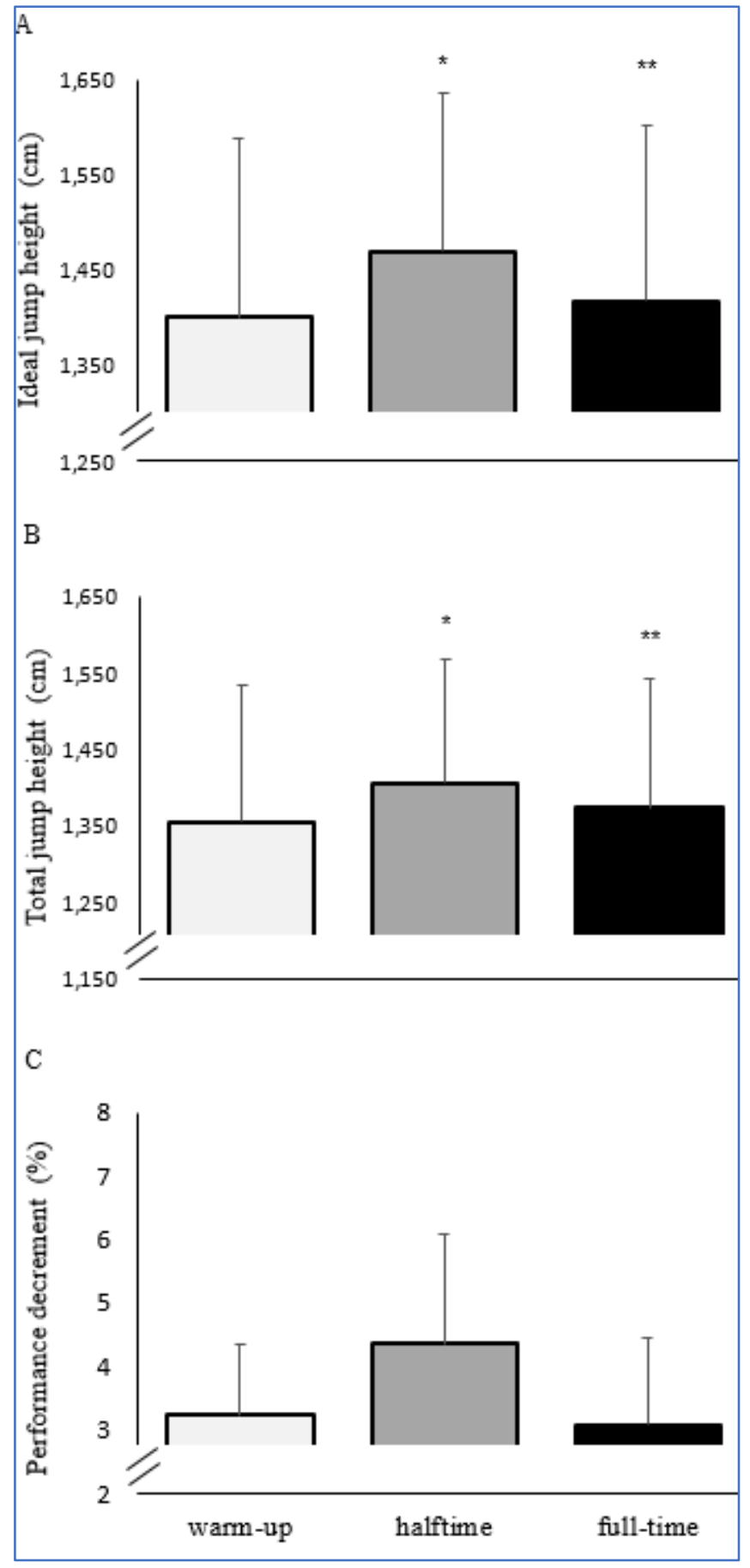

Figure 1. RJT performances indices of the players during the $6 \times 6$ RJT procedure after warm-up, at halftime, and after a full-time basketball game. ${ }^{*} p<0.01$ compared to warm-up; ${ }^{* *} p<0.01$ compared to after halftime.

The significant reduction in the IJ and the TJ performance indices at the end of the game compared to these performance indices at halftime probably demonstrated the accumulative fatigue effect of the full-game activity. Such an accumulative fatigue effect was not seen at halftime compared to the post-warmup RJT. A relative reduction, although not significant, in performance indices following the full-game RST compared to the halftime performance indices, was also seen in the Meckel et al. [8] study. Although there are no experimental evidence available, it has been speculated that higher core temperatures, decreased muscle glycogen and increased lipid metabolism can contribute to this fatigue [14].

The present study also examined the relation between aerobic capacity of the player and RJT performance indices at the various stages. In that context, it has been suggested that high aerobic fitness level is required for increased anaerobic performance during sustained intermittent activities [15]. This conclusion is based on the fact that the re-synthesis of PC (creatine phosphate) is primarily due to oxidative processes [16]. However, there were inconsistent results from correlation analysis between aerobic markers and repeated activity performance indicators, and while some studies showed substantial correlations between the two $[10,11]$, others found them not to be correlated [17]. No significant correlations were found in the present study between any of the RJT performances indices and the aerobic capacity at all of the game stages. These findings may indicate that repeated jumping activity does not profoundly rely or depend on the aerobic system as the main energy supplier for an activity of this nature among young basketball players. Support for this assumption was seen in the findings of Meckel et al. [10], who sought to examine the relationship between the aerobic capacity and the RJT and RST performance indices of professional volleyball players. As in the current study's results, no significant relationships were found between the players' aerobic capacity and any of the performance indices of the RJT (e.g. IJ, TJ, or PD) following a standard warm-up. However, significant correlations were found in their study between the aerobic capacity and all the performance indices of an RST (e.g. IT, TT, and PD). The researchers speculated that the RJT imposes a different physiological stress on the working muscles compared to the RST. They proposed that while fatigue in the RST is mainly related to metabolism and $\mathrm{CP}$ replenishment in the activated muscles, fatigue in the RJT is mainly related to neuromuscular system functioning and to the muscles' contraction mechanism.

\section{Conclusions}

The higher RJT performance indices at halftime compared to those following the warm-up procedure may point to a lack of efficiency, indicating a need for a change in the preparation process prior to the beginning of a basketball game. It could be suggested 
that, before competitive basketball games with young players, longer and particularly vigorous short exercises like jumping, sprinting and changes in running direction should be integrated into the warmup protocol. Such warm-up drills may better prepare the neuromuscular and the metabolic systems for the upcoming aggressive, mostly anaerobic-type activity, that is expected to take place during the game.

The fact that there were no significant correlations between the aerobic capacity and any of the RJT performance indices at all the game stages could imply that jump activities do not rely upon or profoundly depend on the aerobic system as a main energy supplier for young basketball players. Coaches and players may therefore consider this when designing a relevant training program - one that probably should prioritize anaerobic-power type training over long and low-intensity aerobic-type training.

\section{References}

[1] N. Ben Abdelkrim, S. El Fazaa, \& J. El Ati, Timemotion analysis and physiological data of elite under-19-year-old basketball players during competition, British Journal of Sports Medicine, 41 (2007) 69-75.

https://doi.org/10.1136/bjsm.2006.032318

[2] M.J. Klusemann, D.B. Pyne, W.G. Hopkins, \& E.J. Drinkwater, Activity profiles and demands of seasonal and tournament basketball competition, International Journal of Applied Exercise Physiology, 8 (2013) 623-629.

https://doi.org/10.1123/ijspp.8.6.623

[3] S.E. Mclnnes, J.S. Carlson, C.J. Jones, \& M.J. McKenna, The physiological load imposed on basketball players during competition, Journal of Sports Sciences, 13 (1995) 387-397.

https://doi.org/10.1080/02640419508732254

[4] L. Torres-Ronda, A. Ric, I. Llabres-Torres, B. de las Heras, \& X.S. Schelling I del Alcazar, Position - dependent cardiovascular response and time motion analysis during training drills and friendly matches in elite male basketball players, The Journal of Strength and Conditioning Research, 30 (2016) 60-70.

https://doi.org/10.1519/JSC. 0000000000001043

[5] A. Scanlan, B. Dascombe, \& P.A. Reaburn, Comparison of the activity demands of elite and sub-elite Australian men's basketball competition, Journal of Sports Science, 29 (2011) 1153-1160. https://doi.org/10.1080/02640414.2011.582509
[6] M. Fitzsimons, B.T. Dawson, \& D. Ward, Cycling and running tests of repeated sprint ability, Australian Journal of Science and Medicine in Sport, 25 (1993) 82-87.

[7] Z. Gharbi, W. Dardouri, R. Haj-Sassi, K. Chamari, \& N. Souissi, Aerobic and anaerobic determinants of repeated sprint ability in team sports athletes, Biology of Sport, 32 (2015) 207-212. https://doi.org/10.5604/20831862.1150302

[8] Y. Meckel, R. Gottlieb, \& A. Eliakim, Repeated sprint tests in young basketball players at different game stages, European Journal of Applied Physiology, 107 (2009) 273-279.

https://doi.org/10.1007/s00421-009-1120-8

[9] G. Ziv, \& R. Lidor, Vertical jump in female and male basketball players - a review of observational and experimental studies, Journal of Science and Medicine in Sport, 13 (2010) 332339.

https://doi.org/10.1016/j.jsams.2009.02.009

[10] Y. Meckel, M. May-rom, A. Ekshtien, D. Nemet, \& A. Eliakim, Relationships among two repeated activity tests and aerobic fitness of volleyball players, The Journal of Strength and Conditioning Research, 29 (2015) 2122-2127. https://doi.org/10.1519/JSC.0000000000000859

[11] Y. Meckel, A. Einy, R. Gottlieb, \& A. Eliakim, Repeated sprint ability in young soccer players at different game stages, The Journal of Strength and Conditioning Research, 28 (2014) 25782584.

https://doi.org/10.1519/JSC.0000000000000383

[12] A. St Clair Gibson, S. Broomhead, M.I Lambert, \& J.A. Hawley, Prediction of maximal oxygen uptake from a 20-m shuttle run as measured directly in runners and squash players, Journal of Sports Science, 16 (1998) 331-335.

https://doi.org/10.1080/02640419808559361

[13] G.A. Borg GA. Psychophysical bases of perceived exertion. Medicine and Science in Sports and Exercise 14 (1982) 377-382.

https://doi.org/10.1249/00005768-198205000$\underline{00012}$

[14] N. Ben Abdelkrim, C. Castagna, I. Jabri, T. Battikh, S. El Fazaa, \& J. El Ati, Activity profile and physiological requirements of junior elite basketball players in relation to aerobicanaerobic fitness, The Journal of Strength and Conditioning Research, 24 (2010) 2330-2342. https://doi.org/10.1519/JSC.0b013e3181e381c1

[15] D.L. Tomlin, \& H.A. Wenger, The relationship between aerobic fitness and recovery from high 
intensity intermittent exercise, Sports Medicine, 31 (2001) 1-11.

https://doi.org/10.2165/00007256-200131010$\underline{00001}$

[16] P.D. Balsom, G.C. Gaitanos, B. Ekblom, \& K. Soderlund, Reduced oxygen availability during high intensity intermittent exercise impairs performance, Acta Physiologica - The Scandinavian physiological society, 152 (1994) 279-285.

https://doi.org/10.1111/j.1748-1716.1994.tb09807.x

[17] G. Wadley, \& P. Le Rossignol, The relationship between repeated sprint ability and the aerobic and anaerobic energy systems, Journal of Science and Medicine in Sport, 1 (1998) 100110.

https://doi.org/10.1016/s1440-2440(98)80018-2

\section{Acknowledgement}

The authors would like to thank each of the participants for their efforts in completing the physical tasks needed for the study. The authors would also like to thank the coaching staff for their cooperation and support in the present study.

\section{Funding}

This study was not funded by any grant

\section{Conflict of interest}

The authors declare that they have no actual or potential conflict of interest, including financial, personal or other relationships with people or organizations that could have inappropriately influenced this work.

\section{Informed consent}

All participants gave written informed consent to participate in this study.

\section{About The License}

(C) The author(s) 2020. The text of this article is open access and licensed under a Creative Commons Attribution 4.0 International License 